\title{
A Rare Case of Recurrent Metastatic Solid Pseudopapillary Neoplasm of the Pancreas
}

Hye Seung Lee · Han Kyeom Kim Bong Kyung Shin · Jin Hyuk Choi Yoo Jin Choi · Ha Yeon Kim

Department of Pathology, Korea University Guro Hospital, Seoul, Korea

Received: March 30, 2016

Revised: May 21, 2016

Accepted: June 16, 2016

\section{Corresponding Author}

Bong Kyung Shin, MD, PhD

Department of Pathology, Korea University Guro

Hospital, Korea University College of Medicine, 148

Gurodong-ro, Guro-gu, Seoul 08308, Korea

Tel: +82-2-2626-1472

Fax: +82-2-2626-1486

E-mail: tabrega@korea.ac.kr
A 61-year-old woman visited our hospital for bilateral multiple lung nodules and a mass in her thorax. She had a long history of multiple metastatic recurrences of solid pseudopapillary neoplasm (SPN); 24 years previously, the patient had undergone pylorus-preserving pancreaticoduodenectomy for a $9.9 \times 8.6 \mathrm{~cm}$ mass in the pancreatic head. The tumor was diagnosed as an SPN. Nine years later, metastatic nodules were found on computed tomography in the patient's liver and peritoneum and were excised. She subsequently underwent an additional eight metastatectomy procedures in diverse organs. For the presented event, the lung nodules were removed. The prevalence of malignant SPN in the general population is 5\%-15\%. However, multiple metastatic recurrence of malignant SPN is rare; the lung is a particularly rare site of metastasis, found in only three cases in the literature. Here, we describe this exceptional case and provide a literature review.

Key Words: Solid pseudopapillary neoplasm; Lung; Neoplasm metastasis
Solid pseudopapillary neoplasm (SPN) of the pancreas is a rare low-grade malignant neoplasm that accounts for $0.9 \%$ $2.7 \%$ of all exocrine pancreatic neoplasms. SPN was first reported by Frantz in $1959^{1,2}$ and has previously been called a Frantz tumor, solid-pseudopapillary tumor, solid-cystic tumor, solid and papillary epithelial neoplasm, and papillary-cystic tumor. These tumors predominantly occur in young women, with a female-to-male ratio of 9:1. ${ }^{1}$ SPN metastasizes in only 5\%$15 \%$ of all cases, and common sites include the liver; portal, splenic, or superior mesenteric vein; spleen; omentum; peritoneum; duodenum; or other organs. ${ }^{1,2}$ The lung is a very rare metastatic site and has only been reported in three other cases in the literature. ${ }^{3-5}$ In this study, we introduce a case of SPN with lung metastasis following 24 years of multiple recurrences.

\section{CASE REPORT}

\section{Clinical summary}

A 61-year-old woman visited Korea University Guro Hospital (KUGH) for bilateral multiple lung nodules and a mass in the intrathoracic soft tissue. Twenty-four years prior, she had pre- sented to KUGH with epigastric pain; computed tomography (CT) found a $9.9 \times 8.6 \mathrm{~cm}$ heterogeneous mass in the pancreatic head. The tumor was removed and histologically diagnosed as SPN. After tumor removal, the patient underwent two choledochojejunostomy procedures but showed no sign of tumor recurrence. Nine years passed before her next visit for this same condition, when some newly developed nodules in her liver and peritoneum were biopsied and confirmed as metastatic SPN. During the next 15 years, the patient suffered eight additional metastatic recurrences in her liver, peritoneum, omentum, spleen, ovary, uterus, colon, mesocolon, and retroperitoneum before finally developing the present multiple lung metastases. A metastatectomy was performed to remove the lung nodules, and the patient is currently alive (Fig. 1). This study was approved by the Institutional Review Board (KUGH 15363-001) of KUGH.

\section{Pathologic findings}

The clinical and pathologic features of the primary tumor were reviewed in the patient's electronic medical record. The glass slides and paraffin blocks that had been made from the recurrent tumors were also available for review. Overall, the tumors 
showed a pseudopapillary pattern with relatively monomorphic nuclei and abundant eosinophilic cytoplasm. However, we noted a certain degree of change over time, as summarized in Table 1. In accordance with the histological changes, we subdivided the lesions into early (first to third operations), intermediate (fourth to seventh), and late (eighth to 11th) lesions. In the early meta-

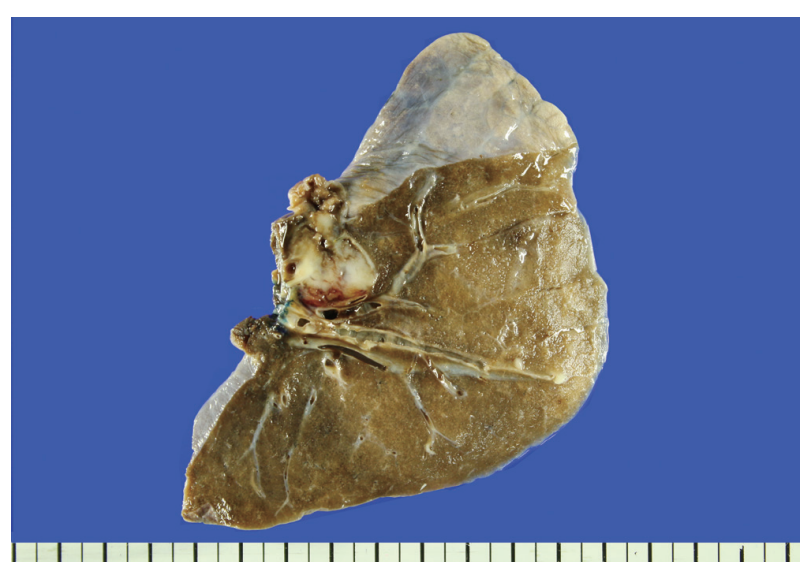

Fig. 1. Gross specimen of the patient's lung metastasis. A solid, well-demarcated and cystic mass with hemorrhage is seen. static lesions, the tumor showed frequent cystic changes with bland nuclei and indistinct necrobiotic nests without capsular invasion. The Ki-67 labeling index was also very low $(<1 \%)$. In the intermediate lesions, however, frequent necrobiotic nests (from the fourth to seventh) and capsular invasions (from the fifth) were noted. The Ki-67 labeling index, which was assessed using an image analysis program, increased to $5 \%$ (sixth). In the late lesions, malignant histological features, such as distinct tumor necrosis beyond necrobiotic nests (from the seventh), peritumoral infiltration of the soft tissue or organ (from the seventh), prominent solid architecture (from the eighth), nuclear atypia (from the ninth), and lymphatic emboli (11th), were seen (Fig. $2 \mathrm{~A}-\mathrm{D})$. The average Ki-67 labeling index increased gradually $(0.03 \%, 2.7 \%$, and $5.6 \%$ in early, intermediate, and late metastatic lesions, respectively). Mitosis was usually absent with exceptions of 5/10 high-power field (HPF) (fifth) and 2/10 HPF (seventh) but did not show a significant change. All of the tumors showed identical immunohistochemical results and were diffusely positive for $\beta$-catenin, vimentin, neuron-specific enolase, CD10, cyclin D1, progesterone receptor, CD56, and synaptophysin. Inter-
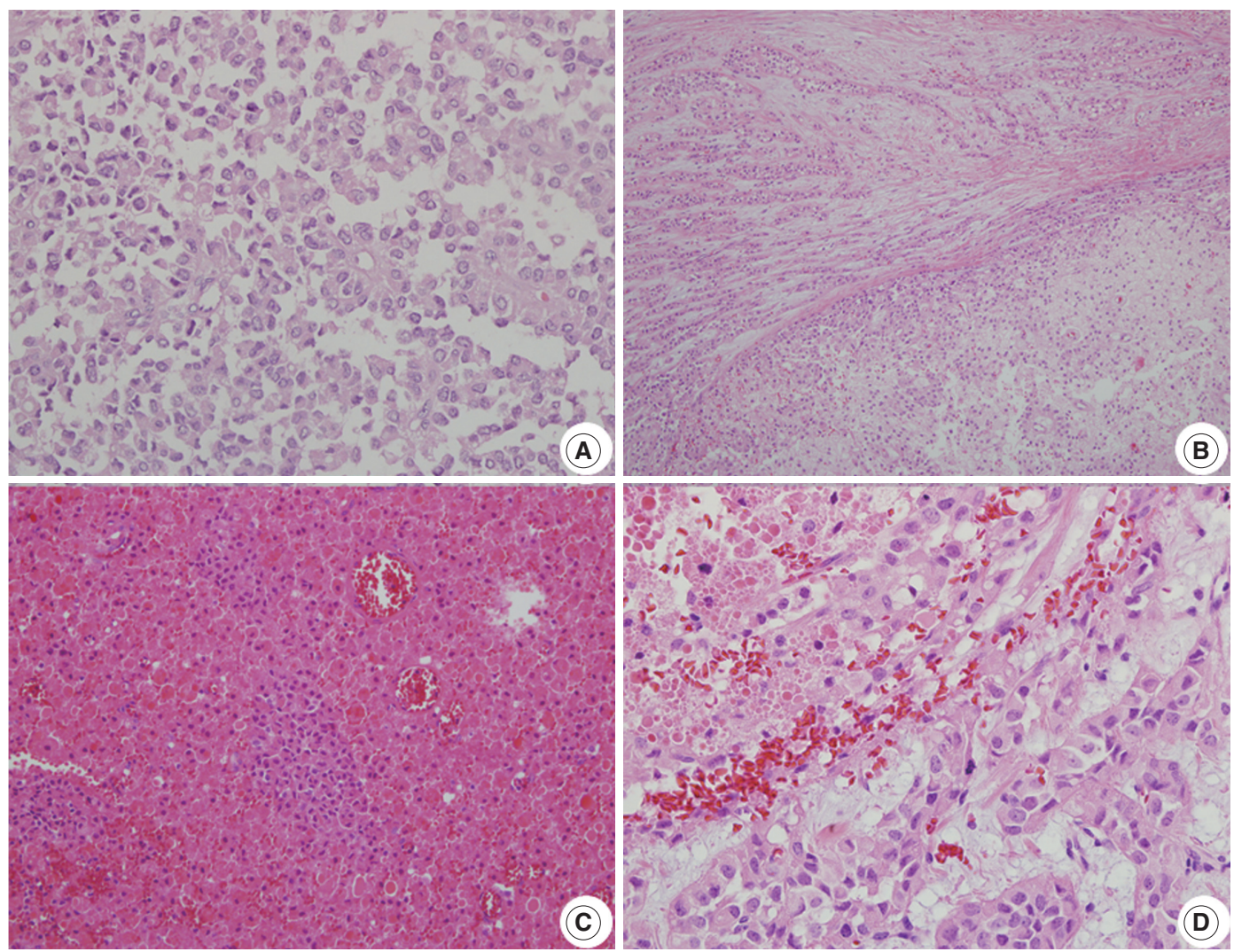

Fig. 2. Microscopic findings. (A) A loosely cohesive pseudopapillary pattern. (B) Capsular invasion with a cord or glandular pattern is visible in the intermediate and late metastatic lesions. (C) Necrobiotic nests are present in the intermediate lesions. (D) Distinct nuclear atypia is seen in the late lesions. 

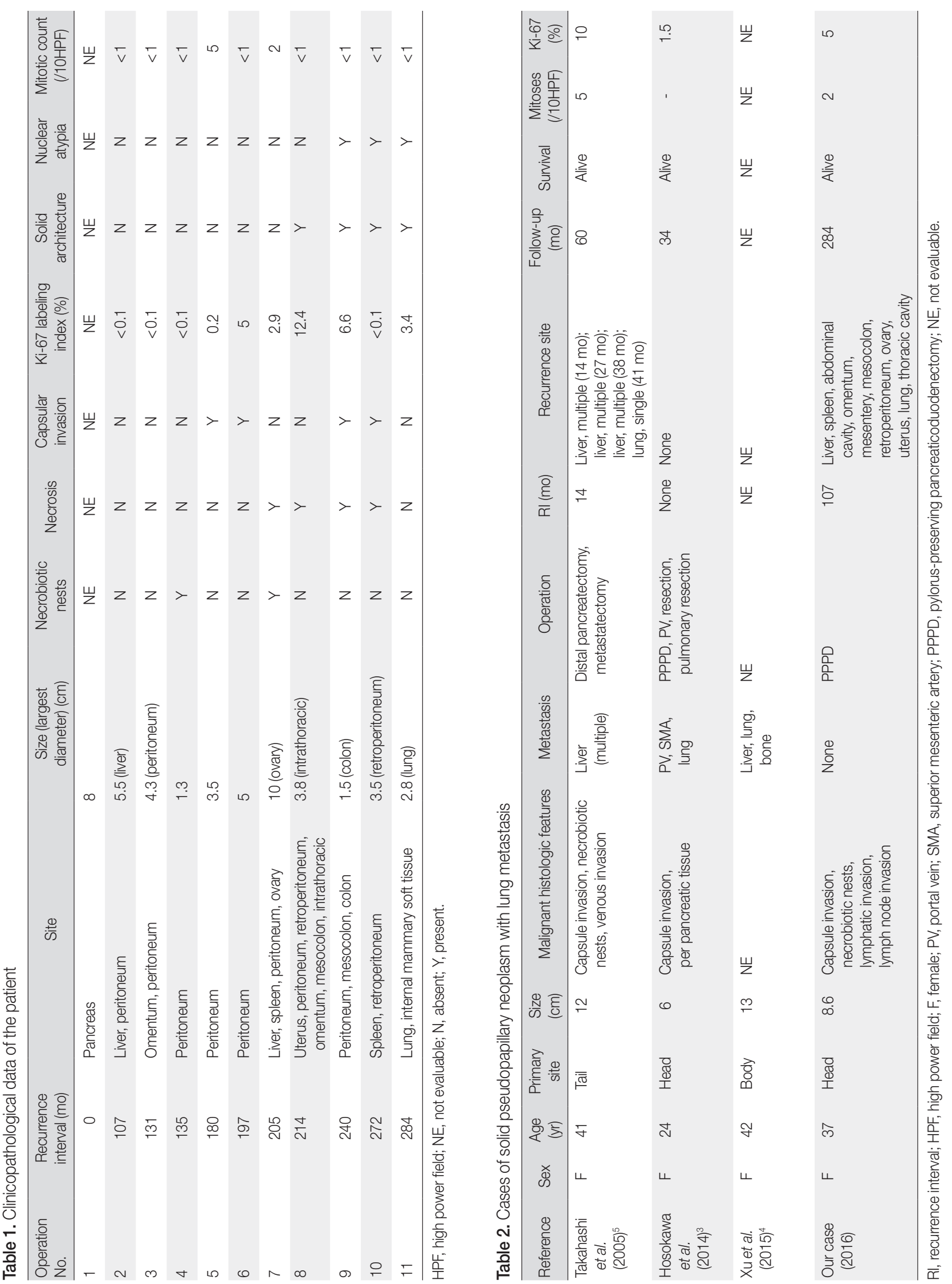
estingly, they were also positive for chromogranin A, which is very rare in SPN.

\section{DISCUSSION}

Generally, the prognosis of SPN is good. Over $95 \%$ of patients with a solitary pancreatic lesion are cured by complete excision. Even with local spread, recurrences, or metastases, long periods of disease-free survival have been recorded. ${ }^{1}$ The general prevalence of metastatic SPN is $5 \%-15 \%,{ }^{1,2}$ Debulking is recommended for these lesions in order to increase the chances of survival. ${ }^{6}$ The lung is a very rare metastatic site and was found in only three cases in the literature.-5 The first was reported in 2005 by Takahashi et al. . $^{5}$ and showed a lung lesion following three liver metastases. Hosokawa $e t a l .3$ reported the second case in 2014, which demonstrated concurrent portal vein and superior mesenteric arterial invasion. The third case was described by $\mathrm{Xu}$ et al. in 2015, ${ }^{4}$ with both liver and bone involvement. Two of the three cases showed a good survival outcome (Table 2). Our case also reveals a long overall survival period despite many recurrences.

After reviewing the eight large studies in the literature, the most commonly quoted malignant SPN characteristics were nuclear atypia (5/8), angioinvasion (5/8), lymph node metastasis (4/8), mitotic count (3/8), advanced age (2/8), size (2/8), capsular invasion (2/8), perineural invasion (2/8), extra-pancreatic invasion (2/8), significant MIB-1 index (2/8), and prominent necrobiotic nests $(1 / 8))^{1,3,5-10}$ Of the four cases with lung metastases (including ours), three showed liver metastasis, three exhibited capsular invasion, and two demonstrated necrobiotic nests (Table 2). Our case initially presented with a bland-looking tumor; however, most of the malignant features including nuclear atypia, lymph node metastasis (upon CT), lymphatic invasion, large tumor size, capsular invasion, and necrobiotic nests developed in later metastases.

Though many studies have claimed that nuclear atypia is a malignant prognostic factor, there is still some debate on this topic. For example, Kim et al. ${ }^{11}$ recently suggested that some subsets of SPN with nuclear pleomorphism exist with stronger p53 immunoreactivity but do not appear to be more aggressive than conventional SPN. The 2010 World Health Organization classification for tumors of the pancreas also claims that nuclear atypia of pancreas SPN cannot predict malignant tumor behavior. ${ }^{12}$ In this study, nuclear atypia accompanied other malignant features. However, due to the patient's relatively good prognosis, the atypia is still a predictor of metastasis but should not be considered a poor prognostic indicator of overall survival. More studies of SPN focusing on nuclear atypia will be needed to further explain this connection.

In conclusion, this case depicts the aggressive progression of tumor cells by repeated peritoneal seeding and lymphatic invasion that still showed an indolent clinical course despite multiple recurrences, including the lung.

\section{Conflicts of Interest}

No potential conflict of interest relevant to this article was reported.

\section{REFERENCES}

1. Papavramidis T, Papavramidis S. Solid pseudopapillary tumors of the pancreas: review of 718 patients reported in English literature. J Am Coll Surg 2005; 200: 965-72.

2. Yu PF, Hu ZH, Wang XB, et al. Solid pseudopapillary tumor of the pancreas: a review of 553 cases in Chinese literature. World J Gastroenterol 2010; 16: 1209-14.

3. Hosokawa I, Shimizu H, Ohtsuka M, et al. Preoperative diagnosis and surgical management for solid pseudopapillary neoplasm of the pancreas. J Hepatobiliary Pancreat Sci 2014; 21: 573-8.

4. Xu M, Shi X, Wan T, et al. Treatment and prognosis of solid pseudopapillary neoplasms with metastases or recurrence. Zhonghua Wai Ke Za Zhi 2015; 53: 685-9.

5. Takahashi Y, Fukusato T, Aita K, et al. Solid pseudopapillary tumor of the pancreas with metastases to the lung and liver. Pathol Int 2005; 55: 792-6.

6. El Nakeeb A, Abdel Wahab M, Elkashef WF, Azer M, Kandil T. Solid pseudopapillary tumour of the pancreas: incidence, prognosis and outcome of surgery (single center experience). Int J Surg 2013; 11: 447-57.

7. Nishihara K, Nagoshi M, Tsuneyoshi M, Yamaguchi K, Hayashi I. Papillary cystic tumors of the pancreas. Assessment of their malignant potential. Cancer 1993; 71: 82-92.

8. Tipton SG, Smyrk TC, Sarr MG, Thompson GB. Malignant potential of solid pseudopapillary neoplasm of the pancreas. Br J Surg 2006; 93: 733-7.

9. Yu P, Cheng X, Du Y, et al. Solid pseudopapillary neoplasms of the pancreas: a 19-year multicenter experience in China. J Gastrointest Surg 2015; 19: 1433-40.

10. Ansari D, Elebro J, Tingstedt B, et al. Single-institution experience with solid pseudopapillary neoplasm of the pancreas. Scand J Gastroenterol 2011; 46: 1492-7. 
11. Kim SA, Kim MS, Kim MS, et al. Pleomorphic solid pseudopapillary neoplasm of the pancreas: degenerative change rather than high-grade malignant potential. Hum Pathol 2014; 45: 166-74.

12. Kloppel G, Hruban RH, Klimstra DS, et al. Solid-pseudopapillary tumor of pancreas. In: Bosman FT, Carneiro F, Hruban RH, Theise ND, eds. World Health Organization classification of tumours of the digestive system. Lyon: IARC Press, 2010; 327-30. 